\title{
Narrow-band anisotropic electronic structure of $\operatorname{ReS}_{2}$
}

\author{
D. Biswas, ${ }^{1}$ Alex M. Ganose, ${ }^{2,3}$ R. Yano,${ }^{4}$ J. M. Riley, ${ }^{1,3}$ L. Bawden, ${ }^{1}$ O. J. Clark, ${ }^{1}$ J. Feng, ${ }^{1}$ L. Collins-Mcintyre, ${ }^{1}$ \\ M. T. Sajjad, ${ }^{5}$ W. Meevasana, ${ }^{6}$ T. K. Kim, ${ }^{3}$ M. Hoesch, ${ }^{3}$ J. E. Rault, ${ }^{7}$ T. Sasagawa, ${ }^{4}$ David O. Scanlon, ${ }^{2,3}$ and P. D. C. King ${ }^{1, *}$ \\ ${ }^{1}$ SUPA, School of Physics and Astronomy, University of St. Andrews, St. Andrews KY16 9SS, United Kingdom \\ ${ }^{2}$ Department of Chemistry, University College London, 20 Gordon Street, London WC1H OAJ, United Kingdom \\ ${ }^{3}$ Diamond Light Source, Harwell Campus, Didcot OX11 ODE, United Kingdom \\ ${ }^{4}$ Materials and Structures Laboratory, Tokyo Institute of Technology, Kanagawa 226-8503, Japan \\ ${ }^{5}$ Organic Semiconductor Centre, SUPA, School of Physics and Astronomy, University of St. Andrews, St. Andrews KY16 9SS, United Kingdom \\ ${ }^{6}$ School of Physics and Center of Excellence on Advanced Functional Materials, Suranaree University of Technology, \\ Nakhon Ratchasima 30000, Thailand \\ ${ }^{7}$ Synchrotron SOLEIL, CNRS-CEA, L'Orme des Merisiers, Saint-Aubin-BP48, 91192 Gif-sur-Yvette, France
}

(Received 14 March 2017; revised manuscript received 16 June 2017; published 16 August 2017)

\begin{abstract}
We have used angle-resolved photoemission spectroscopy to investigate the band structure of $\mathrm{ReS}_{2}$, a transitionmetal dichalcogenide semiconductor with a distorted 1T crystal structure. We find a large number of narrow valence bands, which we attribute to the combined influence of structural distortion and spin-orbit coupling. We further show how this leads to a strong in-plane anisotropy of the electronic structure, with quasi-one-dimensional bands reflecting predominant hopping along zigzag Re chains. We find that this does not persist up to the top of the valence band, where a more three-dimensional character is recovered with the fundamental band gap located away from the Brillouin zone center along $k_{z}$. These experiments are in good agreement with our density-functional theory calculations, shedding light on the bulk electronic structure of $\mathrm{ReS}_{2}$, and how it can be expected to evolve when thinned to a single layer.
\end{abstract}

DOI: 10.1103/PhysRevB.96.085205

\section{INTRODUCTION}

The semiconducting transition-metal dichalcogenides (TMDCs) with the formula $M X_{2}$ (where $M$ represents transition metal and $X$ represents chalcogen) have recently attracted a great deal of attention for their intriguing optical and electronic properties [1]. These are typically found to depend sensitively on material thickness, where thinning down prototypical compounds such as $\mathrm{MoS}_{2}$ to a single layer drives a crossover from an indirect- to a direct-band-gap semiconductor, accompanied by a dramatic increase in the photoluminescence yield [2-4]. In contrast, recent studies of the group VII TMDCs $\operatorname{Re} X_{2}(X=\mathrm{Se}, \mathrm{S})$ revealed a striking resilience of their measured photoluminescence to changing material thickness [5,6], attributed to a particularly weak interlayer coupling leading to a monolayer-like electronic structure in the bulk. This opens the exciting prospect to achieve optoelectronic functionality from bulk $\operatorname{Re} X_{2}$ of a form that can only be realized by complex fabrication of single-layer samples and devices in group VI semiconducting TMDCs. Coupled with a pronounced anisotropy in their measured optical and electrical properties [7-10], $\operatorname{Re} X_{2}$ materials are therefore important compounds for expanding the functionality of the TMDC class, and they have potential for next-generation technologies [11,12]. The electronic structure underpinning their striking optoelectronic properties, however, remains almost completely unexplored experimentally to date.

The crystal structure of $\operatorname{ReS}_{2}$ (space group: $P \overline{1}$ ) is shown in Fig. 1. This can be described via a structural distortion away from the $1 \mathrm{~T}$ structure that is found for some group $\mathrm{V}$ TMDCs such as $\mathrm{TaS}_{2}$ and group X TMDCs such as $\mathrm{PdTe}_{2}$.

\footnotetext{
*philip.king@st-andrews.ac.uk
}

In the undistorted structure, the transition metal sits at the center of edge-sharing octahedra formed by the chalcogen atoms [Fig. 1(a)], with trigonal antiprismatic point-group symmetry of the transition-metal site [13]. A Jahn-Tellerlike structural distortion causes the formation of zigzag $\mathrm{Re}$ chainlike structures as well as a pronounced out-of-plane buckling of the chalcogen layers [Fig. 1(b)] [5,14]. The in-plane unit vectors are each approximately doubled, and the threefold rotational symmetry of the parent $1 \mathrm{~T}$ structure is lost. While the surface Brillouin zone remains almost hexagonal, here we show how the electronic structure becomes strongly anisotropic along different in-plane directions, with stripelike band contours observed in angle-resolved photoemission (ARPES) measurements of the valence-band constant energy contours [Fig. 1(c)]. We will discuss this anisotropy in detail below, and we show how it exhibits a striking binding-energy dependence tied to the underlying orbital character of the electronic states.

\section{METHODS}

Single crystals of $\mathrm{ReS}_{2}$ were grown by the chemical vapor transport technique with iodine as a transport agent. First, polycrystalline $\mathrm{ReS}_{2}$ was prepared from a mixture of Re and S elements by the solid-state reaction at $900{ }^{\circ} \mathrm{C}$ for $30 \mathrm{~h}$. The obtained $\mathrm{ReS}_{2}$ powder and iodine were sealed in an evacuated quartz tube, and they were heated using a two-heating zone furnace $\left(1050{ }^{\circ} \mathrm{C} / 950{ }^{\circ} \mathrm{C}\right)$ for $150 \mathrm{~h}$. Finally, the obtained crystals were cleaned by rinsing with acetone. This resulted in thin and flat crystals with reflective surfaces. They were found to be insulating from resistivity measurements, while optical transmission measurements indicate a pronounced increase in optical absorption at an energy of $1.47 \pm 0.03 \mathrm{eV}$, consistent with previous studies of the onset of direct optical absorption 
(a) $1 \mathrm{~T}$ structure
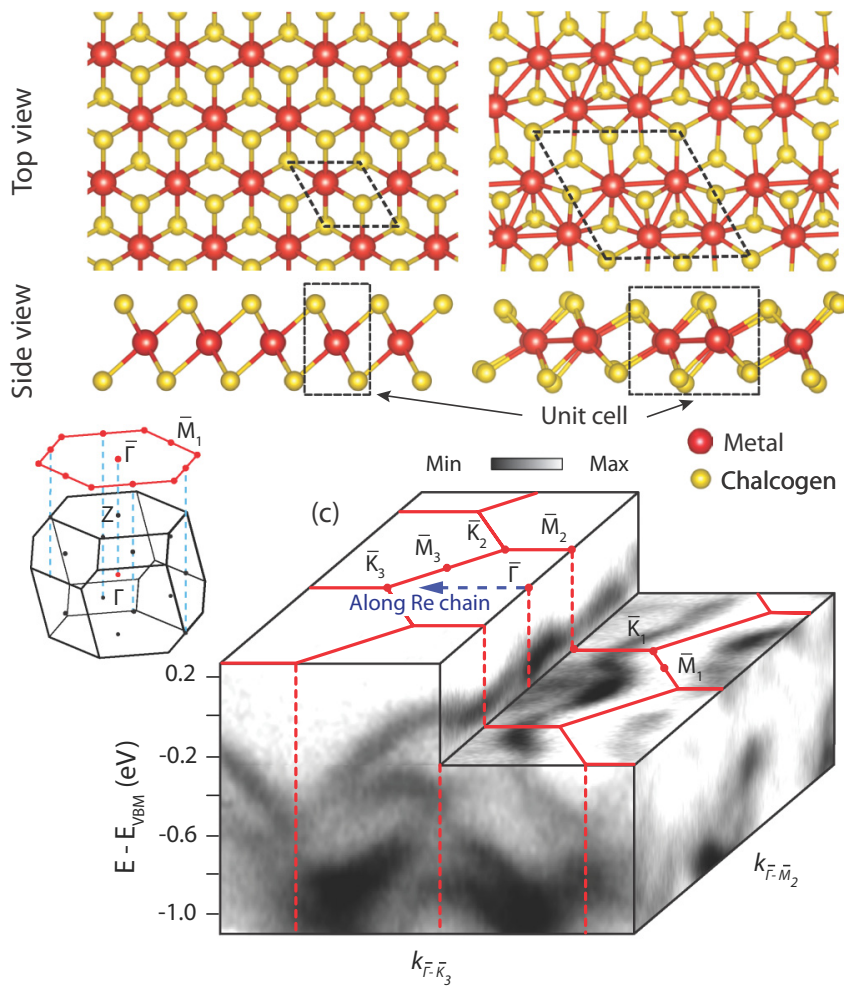

FIG. 1. (a,b) Top and side views of a single layer (one unit cell in height) of an (a) undistorted and (b) distorted 1T crystal structure, respectively. (c) Overview of the valence-band structure as measured by ARPES, showing strong in-plane anisotropy. The surface Brillouin zone is shown as red lines, and the momentum space direction corresponding to the real-space direction along the Re chains is also indicated. The bulk and projected surface Brillouin zones are shown in the inset.

in this material $[15,16]$. Angle-dependent Raman scattering measurements of these samples show strong anisotropy in the $214 \mathrm{~cm}^{-1}$ peak intensity with clear twofold symmetry, as seen in recent reports $[9,17,18]$.

ARPES measurements were performed using the I05 beamline of Diamond Light Source, UK and the CASSIOPEE beamline of SOLEIL synchrotron, France. Samples were cleaved in situ at a measurement temperature between 10 and $20 \mathrm{~K}$, and all measurements were performed using Scienta R4000 hemispherical electron analyzers. From our photonenergy-dependent measurements (Fig. 3), we estimate an inner potential $V_{0}=12 \mathrm{eV}$ employing a free-electron final-state model, similar to the value found in other TMDCs [19-22]. Due to the insulating nature of our samples, charging was evident as a shift of the spectral features to lower kinetic energy. We therefore reference our spectra to the valence-band maximum (VBM), and we compensate for relative charging shifts between spectra (e.g., when changing photon energy) by the relative shift of the $\operatorname{Re} 4 f$ core-level peak. Surface doping was achieved by evaporating $\mathrm{Rb}$ on the sample at the measurement temperature from a well-outgassed SAES alkali-metal source. We predominantly describe the electronic structure as measured within the almost hexagonal surface
Brillouin zone, although the structural distortion causes neighboring corners (face centers) of the surface Brillouin zone to become inequivalent. We thus label these with the conventional $\overline{\mathrm{K}}(\overline{\mathrm{M}})$ notation, with an additional subscript to distinguish inequivalent symmetry points. The Re-Re zigzag chains are oriented perpendicular to the $\bar{\Gamma}-\bar{M}_{2}$ direction in our nomenclature.

All calculations were performed using the Vienna $a b$ initio Simulation Package (VASP) [23-26], a periodic plane-wave DFT code. The projector augmented wave (PAW) method was used to describe the interactions between core and valence electrons [27]. Convergence with respect to the plane-wave basis set and $k$-point sampling was performed, with a cutoff energy of $500 \mathrm{eV}$ and a $k$-point grid of $\Gamma$-centered $4 \times 4 \times 4$ found to be sufficient for the 12-atom unit cell of $\mathrm{ReS}_{2}$. Geometry optimizations were performed using the PBEsol functional [28], a version of the Perdew, Burke, and Ernzerhof (PBE) functional [29] revised for solids. PBEsol has previously been shown to adequately account for weakly dispersive interactions, such as those seen in the layered structure of $\mathrm{ReS}_{2}$, without the need for an additional correction [30,31]. Optimizations were deemed converged when the sum of all forces on each atom totaled less than $10 \mathrm{meV}^{-1}$. The calculated structure parameters $(a=6.424 \AA, b=6.490 \AA$, $c=6.407 \AA, \alpha=106.4^{\circ}, \beta=88.2^{\circ}$, and $\left.\gamma=121.4^{\circ}\right)$ show good agreement with experiment (all within $0.6 \%$ ). To provide an accurate description of the electronic structure of $\mathrm{ReS}_{2}$, the hybrid functional, HSE06 [32], was employed for bandstructure and density-of-states calculations. HSE06 combines $75 \%$ exchange and $100 \%$ of the correlation energies from PBE together with $25 \%$ exact Hartree-Fock (HF) exchange at short ranges, and it has been shown to perform well for a wide range of solid-state semiconductors [33,34]. Special attention was paid to accurately modeling the relativistic effects seen in $\mathrm{Re}$ through use of scalar relativistic PAW pseudopotentials and explicit treatment spin-orbit coupling effects [35].

\section{RESULTS AND DISCUSSIONS}

We start by considering the detailed valence-band dispersions measured along different high-symmetry directions of the surface Brillouin zone (Fig. 2). At the photon energy used, these measurements probe a $\Gamma$ point of the bulk Brillouin zone for $k_{\|}=0$, although we note that we have a finite $k_{z}$-resolution that we estimate as $0.17 \AA^{-1}$ due to the surface sensitivity of photoemission, while the value of $k_{z}$ probed also decreases with increasing in-plane momentum [36]. For the measurements shown here, the value of $k_{z}$ typically varies by less than $10 \%$ of the Brillouin zone height with varying in-plane momentum across the first surface Brillouin zone.

It is immediately evident that there are a large number of bands within $\sim 3 \mathrm{eV}$ of the valence-band top, each with a narrow bandwidth typically on the order of only $200 \mathrm{meV}$. This initially appears counterintuitive, given the spatially extended nature of the Re $5 d$ and $\mathrm{S} 3 p$ orbitals from which these valence states derive. However, the doubled unit-cell dimensions along both in-plane directions would cause a backfolding of the broad bands that would be expected in the undistorted 1T crystal structure. This will drive multiple band crossings 


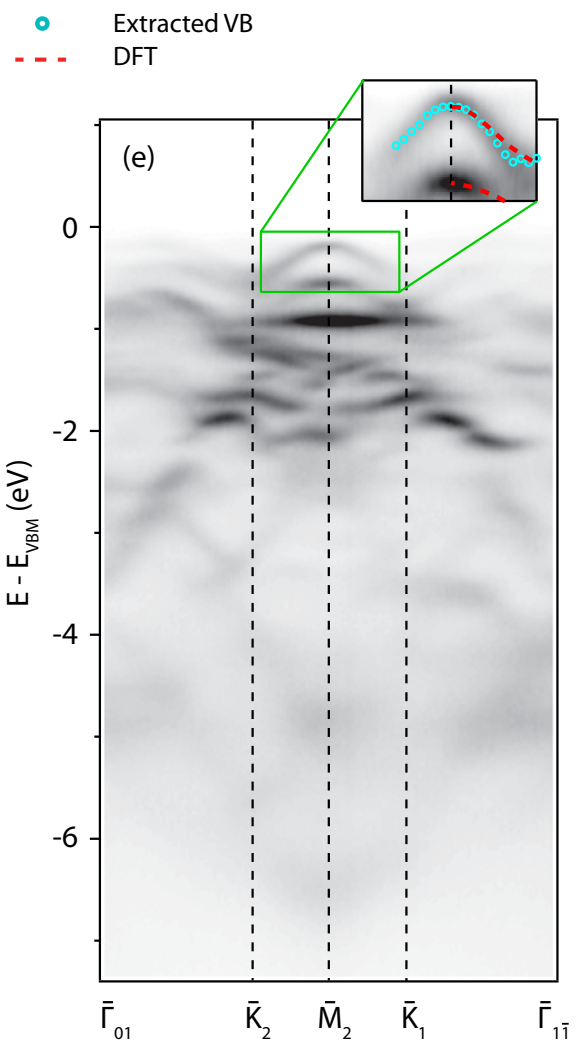

FIG. 2. ARPES measurements $\left(h v=86 \mathrm{eV}\right.$, chosen to probe a bulk $\Gamma$ point along $k_{z}$ ) measured along the (a) $\bar{\Gamma}-\overline{\mathrm{K}}_{3}$, (b) $\bar{\Gamma}-\overline{\mathrm{M}}_{2}$, and (c) $\bar{\Gamma}-\bar{M}_{3}$ directions of the surface Brillouin zone. These cut at different directions to the elongated contours visible in constant energy contours shown in (d) $230 \mathrm{meV}$ below the VBM. The high-symmetry points are labeled in the panel. (e) Equivalent ARPES dispersions, shown over an extended binding-energy range, measured along the boundary of the surface Brillouin zone $\left(\overline{\mathrm{K}}_{2}-\overline{\mathrm{M}}_{2}-\overline{\mathrm{K}}_{1}\right)$. For all measurements, the detailed dispersion of the uppermost valence band is shown in the inset, extracted from fits to EDCs and shown together with corresponding calculated valence-band dispersions from density-functional theory.

throughout the Brillouin zone. Strong spin-orbit coupling can be expected to open pronounced hybridization gaps at these band crossings, thereby creating much narrower bandwidths than would naively be expected considering the undistorted crystal structure. Indeed, tracing the dominant spectral weight, which normally follows the unreconstructed band dispersion in ARPES spectra [37,38], reveals much broader bandwidths from which these narrow bands ultimately result. This is particularly evident along the $\bar{\Gamma}_{01}-\bar{\Gamma}_{1 \overline{1}}$ direction [Fig. 2(e)], which would be a $\overline{\mathrm{K}}-\overline{\mathrm{K}}$ direction in the unreconstructed Brillouin zone, where broad-band-like variations in the spectral weight can be traced dispersing over several electronvolts width. We thus attribute the presence of multiple bands with narrow bandwidths here to the combined effects of structural distortion and strong spin-orbit coupling characteristic of the Re $d$-orbital manifold. A similar mechanism for narrow-band formation was recently proposed in the strong spin-orbit semimetal $\mathrm{SrIrO}_{3}$ [39].

Even within these narrow bands, we find significant differences in bandwidth along different momentum directions. In Figs. 2(a)-2(c), we show the valence-band dispersions measured along $\bar{\Gamma}-\overline{\mathrm{K}}_{3}, \bar{\Gamma}-\overline{\mathrm{M}}_{2}$, and $\bar{\Gamma}-\overline{\mathrm{M}}_{3}$ of the surface Brillouin zone, respectively. Focusing on the uppermost valence band, we find the most dispersive states along $\bar{\Gamma}-\bar{K}_{3}$ [and parallel to this, along $\overline{\mathrm{K}}_{2}-\overline{\mathrm{M}}_{2}-\overline{\mathrm{K}}_{1}$, Fig. 2(e)]. This corresponds to the direction along the Re chains formed by the structural distortion [Fig. 1(b)]. In a tight-binding picture, hopping should therefore be maximal along this direction, leading to the most dispersive states. Even along this direction, however, the valence band at $\Gamma$ is not parabolic, instead flattening over to form a slightly "M"-shaped dispersion. This is evident in fits to our measured energy-distribution curves (EDCs), as well as our density-functional theory calculations, which are in good agreement with our measured dispersions [Fig. 2(a), inset]. Similar M-shaped dispersions have been observed in two-dimensional monochalcogenide semiconductors such as $\mathrm{GaS}$ and $\mathrm{GaSe}$, and found to arise from interorbital interactions $[40]^{1}$. This will lead to an enhanced density of states near the band edge, helping to explain the strong optical absorption and photoluminescence that this compound is known to possess [5]. Upon moving away from this direction, this band top gradually flattens [Fig. 2(c)] before becoming almost nondispersive along the $\bar{\Gamma}-\bar{M}_{2}$ direction (normal to the chain). This points to a limited hopping between the chains, and thus a highly anisotropic in-plane electronic structure. This is also

\footnotetext{
${ }^{1}$ We note that experimentally (Fig. 4), and in our calculations, these $\mathrm{M}$-shaped dispersions are found in the $k_{z}=0$ plane, but not for the in-plane dispersions at the Brillouin zone face along $k_{z}$.
} 
(a)


(d)



(b)
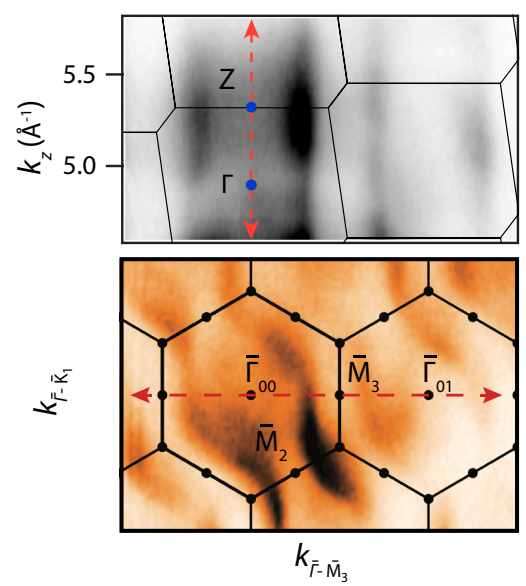

(e) EDC at $\bar{\Gamma}_{00}$ (f) (c)


(g)
Max

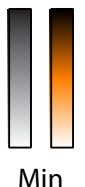

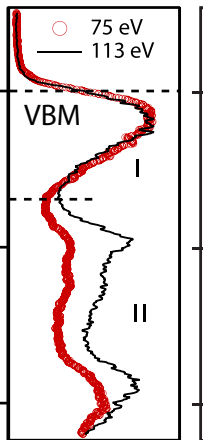

Norm. Int.

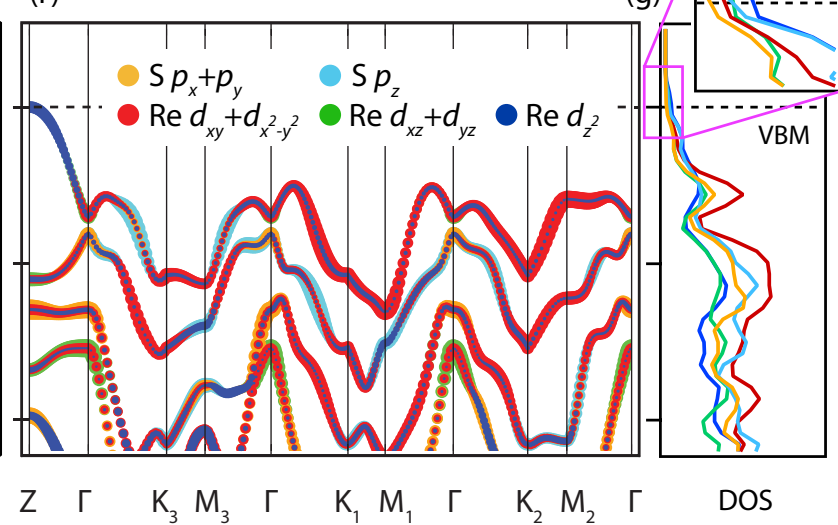

FIG. 3. (a)-(c) $k_{z}$ dependence of the MDC passing through $\bar{\Gamma}_{00}$ and $\bar{\Gamma}_{01}$ (top) and in-plane constant energy contours at a photon energy $h v=86 \mathrm{eV}$, corresponding to $k_{z} \sim 10 \pi / c$, (a) at the valence-band top and (b) $0.3 \mathrm{eV}$ and (c) $0.9 \mathrm{eV}$ below the VBM, respectively, as shown by the blue lines in the dispersion $\left(h v=75 \mathrm{eV}\right.$ ) shown in (d). (e) Comparison of energy-dispersive curves (EDCs) at $k_{\|}=0$ taken using 75 and $113 \mathrm{eV}$ photons, showing an enhanced Re-derived orbital character closer to the band top. (f), $(\mathrm{g})$ Corresponding orbitally resolved calculations from density-functional theory.

evident from a constant energy contour shown $230 \mathrm{meV}$ below the valence-band top in Fig. 2(d), which exhibits band contours that are closed along the $\bar{\Gamma}-\overline{\mathrm{K}}_{3}$ direction but open along the orthogonal $\bar{\Gamma}-\overline{\mathrm{M}}_{2}$ direction. This directly underpins the strong anisotropy of in-plane transport of this compound [8].

It is thought that the coupling strength between the layers of $\mathrm{ReS}_{2}$ is very weak [5]. The electronic states, therefore, would naturally be expected to be predominantly confined within single S-Re-S layers, which, coupled with their strongly anisotropic in-plane dispersion, would result in quasione-dimensional electronic states. In contrast, our photon energy-dependent ARPES measurements (Fig. 3) indicate that this is only true within limited binding-energy ranges. Figures 3(a)-3(c) show the dispersion of electronic states along $\bar{\Gamma}_{00}-\bar{\Gamma}_{01}$ as a function of $k_{z}$ (top) and for varying in-plane momentum for approximately fixed $k_{z}=10 \pi / c \quad(h v=86$ $\mathrm{eV}$, bottom). These are shown at the valence-band maximum [VBM, Fig. 3(a)] and 300 and $900 \mathrm{meV}$ below the VBM, respectively [Figs. 3(b) and 3(c)]. Focusing first at the VBM [Fig. 3(a)], rather than being quasi-one-dimensional, we find that the electronic bands are in fact quasi-three-dimensional. Elongated ellipses are found along both the out-of-plane and in-plane momentum directions, but both form closed contours located around the $\mathrm{Z}$ point of the bulk Brillouin zone (BZ). We therefore find that the VBM is not located at the BZ center, but rather at the $\mathrm{BZ}$ face along $k_{z}$.

In contrast, only $300 \mathrm{meV}$ below the VBM, the valenceband states exhibit only very little out-of-plane dispersion, as is evident from the almost invariant band momenta with varying photon energy [Fig. 3(b)]. By this binding energy, they have also developed the open contours within the transitionmetal plane as discussed above, and so their electronic states can best be described as quasi-one-dimensional in this energy range. At $900 \mathrm{meV}$ below the VBM, the band contours reach close to the BZ boundary within the plane, and a complex band structure is evident along $k_{z}$, with several bands showing significant $k_{z}$ dispersion. Our measurements, therefore, reveal a rich binding-energy dependence of the dimensionality of the electronic structure of $\mathrm{ReS}_{2}$.

We show below how this arises due to a variation in the orbital character of the valence bands. Figures 3(f) and 3(g) show the orbitally projected electronic structure calculated from DFT. While the orbital character is strongly mixed throughout the valence bands, our calculations indicate that 
out-of-plane $\operatorname{Re} 5 d_{3 z^{2}-r^{2}}$ orbitals contribute a significant weight close to the VBM. These out-of-plane orbitals are hybridized with $\mathrm{S} p_{z}$ orbitals and mediate significant interlayer hopping, consistent with the quasi-three-dimensional nature of the electronic states at the VBM. In contrast, in-plane $d$ orbitals dominate slightly ( $\gtrsim 250 \mathrm{meV}$ ) below the VBM, leading to the quasi-one-dimensional dispersions observed above. Deeper still, we find a more significant contribution of S $3 p$ orbitals. Located on either side of the van der Waals gap, these naturally mediate a more significant interlayer hopping than the Re-derived states, explaining the reentrant three-dimensionality observed in our experiment.

This is entirely consistent with our photon-energydependent ARPES data. We show in Fig. 3(e) two EDCs measured at the $\bar{\Gamma}$ point using photon energies of 75 and $113 \mathrm{eV}$. These are selected to probe the same point in $k_{z}$ (the midpoint along $\Gamma-\mathrm{Z}$ ) but to have a different photoionisation cross section of Re $5 d$ and $\mathrm{S} 3 p$ states, with the former photon energy almost three times more sensitive to Re- versus S-derived states than the latter [41]. A significantly greater relative spectral weight more than $\sim 350 \mathrm{meV}$ below the VBM for measurements at $h v=113 \mathrm{eV}$ points to a much larger $\mathrm{S} 3 p$-derived character of the valence-band states here than at the VBM, as found in our calculations. Together, these measurements and calculations reveal that the dimensionality of electronic states in $\mathrm{ReS}_{2}$ is inherently tied to their varying orbital makeup.

A key question across the TMDC materials class has been the nature and dimensional control of the fundamental band gap. To address this here, we have electron-doped the surface of $\mathrm{ReS}_{2}$ via deposition of small concentrations of $\mathrm{Rb}$ atoms in order to populate the conduction-band states. Our measured ARPES spectra from as-cleaved and Rb-doped samples are shown in Figs. 4(a) and 4(b), respectively. We find that new states are populated $\sim 1.2 \mathrm{eV}$ above the VBM, located at the $\bar{\Gamma}$ point of the surface Brillouin zone. We thus attribute these as occupied conduction-band states. Our photon-energy-dependent ARPES data [Figs. 4(d) and 4(e)] indicate that not only the VBM but also the conduction-band minimum $(\mathrm{CBM})$ has a significant dispersion along $k_{z}$. The conduction and valence bands disperse in the opposite sense, ensuring that a direct band gap with high joint density of states persists in bulk $\mathrm{ReS}_{2}$, albeit located at the BZ boundary along $k_{z}$ rather than at the $\mathrm{BZ}$ center. This is supported by our DFT calculations [Fig. 4(f)]. We note that the out-of-plane dispersion is slightly overestimated by these calculations, suggesting too high an interlayer interaction in our theory. Nonetheless, this indicates that a three-dimensional dispersion at the valence-band top is inherent to the ideal crystal structure of $\mathrm{ReS}_{2}$, and not driven by enhanced interlayer hopping via, e.g., stacking faults or intercalated transport agents from the growth. Indeed, our calculations are in excellent qualitative agreement with our experimental measurements of a direct band gap at the $\mathrm{Z}$ point of the bulk Brillouin zone.

We note from the measurements and calculations presented above that we cannot fully exclude a slightly smaller indirect band gap located away from high-symmetry lines. The nature of the optical band gap in $\mathrm{ReS}_{2}$ has proved controversial, with an indirect band gap suggested by recent optical measurements [42]. To investigate this in more detail, we have performed
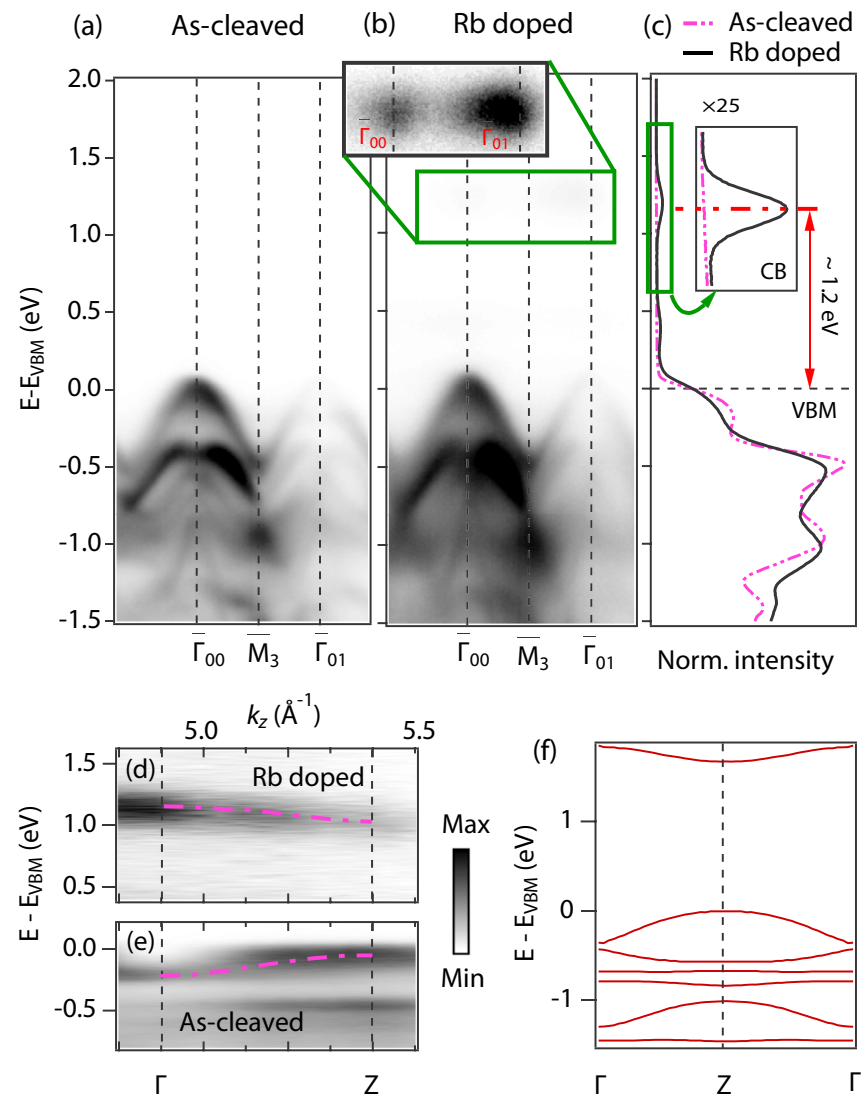

FIG. 4. (a) and (b) ARPES spectra for as-cleaved and Rb-doped $\mathrm{ReS}_{2}$ along $\bar{\Gamma}-\overline{\mathrm{M}}_{3}$. The inset shows the energy region around the conduction-band states in higher contrast. (c) Integrated spectra of the measurements shown in $(a, b)$ for the as-cleaved and doped sample, again showing the filling of the conduction band. (d) and (e) $k_{z}$ dispersion measured at $k_{\|}=0$ for doped and clean $\operatorname{ReS}_{2}$. (f) Calculated $k_{z}$ dispersion along $\Gamma$-Z from DFT.

a set of calculations with an extremely dense $k$ mesh. To make this computationally feasible, we utilized the PBEsol functional. At this level of theory, we indeed find that the VBM is shifted away from the high-symmetry line, with the true VBM located at a $k$-point of $k_{v}^{1}=(0.20,-0.20,0.27)$. This is located only $28 \mathrm{meV}$ higher in energy than the calculated valence-band eigenstates at the $\mathrm{Z}$ point. Moreover, when calculating the electronic structure at $k_{v}^{1}$ with the more accurate, but computationally expensive, HSE06+SOC functional used for the rest of this work, we found that the valence band at $\mathrm{Z}$ remained $77 \mathrm{meV}$ higher in energy. Therefore, with the highest level of theory, $\operatorname{ReS}_{2}$ remains a direct-band-gap semiconductor, with the direct band gap located at the $\mathrm{Z}$ point of the Brillouin zone. It seems likely, however, that there are nearly degenerate optical transitions at other momentum points.

Intriguingly, the band gap we extract from our ARPES measurements $(\sim 1.2 \mathrm{eV})$ is $\sim 300 \mathrm{meV}$ smaller than the measured optical band gap. The surface doping approach used here could be expected to create a near-surface downward band bending, rather than inducing a rigid band filling of the conduction-band states [43-46]. This, however, would be expected to lead to an increased band gap via quantum 
confinement of the conduction-band states, and it is inconsistent with the three-dimensional dispersions of the conductionband states that we observe. The smaller band gap observed here may instead indicate an increased electronic screening due to the high near-surface electron density, creating a strong renormalization of the electronic band gap from its value in the undoped semiconductor, as has been observed in other TMDC compounds $[47,48]$. This possibility requires further exploration, both experimentally and theoretically, but it may point to the presence of rather strongly bound excitons even in bulk $\operatorname{ReS}_{2}$. This would seem broadly consistent with the observed pronounced excitonic features in optical spectra $[5,42]$.

\section{CONCLUSION}

In conclusion, our study has revealed the bulk electronic structure of the TMDC semiconductor $\operatorname{ReS}_{2}$. We have imaged how the presence of in-plane structural distortion leading to the formation of Re zigzag chains, combined with weak interlayer coupling in this compound, drives the formation of quasi-one-dimensional electronic states in its valence bands, underpinning its anisotropic optoelectronic properties. However, we have also shown how this breaks down both near the band edges and at higher binding energies, where a more significant three-dimensionality of the electronic states results due to the mixing in of atomic orbitals with out-of-plane character. The resulting electronic structure still leads to a direct or nearly direct band-gap semiconductor in bulk, unlike for other bulk TMDCs such as $\mathrm{MoS}_{2}$, but with the band extrema located away from the Brillouin zone center along $k_{z}$. The $k_{z}$ dispersion of the band-edge states naturally explains the blueshift observed in the peak energy of photoluminescence when $\mathrm{ReS}_{2}$ is thinned to a single monolayer [5], as quantum confinement in the $z$ direction will increase the energy of the direct band gap in this compound. Together, this opens exciting new possibilities for tuning and utilizing the anisotropic electrical and optical response of $\mathrm{ReS}_{2}$ bulk and thin-film samples.
Note added. While finalizing our work, a paper appeared on arXiv that also reported a non-negligible out-of-plane dispersion of $\mathrm{ReS}_{2}$ [49]. Their data seem to be consistent with ours.

\section{ACKNOWLEDGMENTS}

We are grateful to Daniel Wolverson, University of Bath, UK, for discussing the results of their similar measurements with us prior to publication, which also appear to be consistent with the data presented here [50]. We gratefully acknowledge F. Bertran and P. Le Fèvre for ongoing technical support of the CASIOPEE beam line at SOLEIL. This work was supported by the Engineering and Physical Sciences Research Council, UK (Grant No. EP/M023427/1). This work also made use of the ARCHER UK National Supercomputing Service (http://www.archer.ac.uk), via membership of the UK's HEC Materials Chemistry Consortium, which is funded by EPSRC (EP/L000202). We thank Diamond Light Source (via Proposals No. SI9500 and No. SI11383) and SOLEIL synchrotrons for access to Beamlines I05 and CASSIOPEE, respectively, which contributed to the results presented here. A.M.G. acknowledges Diamond Light Source for the cosponsorship of a studentship on the EPSRC Centre for Doctoral Training in Molecular Modelling and Materials Science (EP/L015862/1). J.M.R., L.B., and O.J.C. acknowledge EPSRC for Ph.D. studentship funding through Grants No. EP/L505079/1, No. EP/G03673X/1, and No. EP/K503162/1, respectively. W.M. received support from Thailand Research Fund and Suranaree University of Technology (Grant No. BRG5880010). T.S. was supported by a CREST project from Japan Science and Technology Agency (JST) and a Grants-in-Aid for Scientific Research (B) (16H03847) from Japan Society for the Promotion of Science (JSPS). D.O.S. acknowledges support from the EPSRC (EP/N01572X/1). D.O.S. and P.D.C.K. acknowledge membership of the Materials Design Network. Data underpinning this publication can be accessed at http://dx.doi.org/10.17630/6619d537-c731-48b885e3-cfb863244f96.
[1] Q. H. Wang, K. Kalantar-Zadeh, A. Kis, J. N. Coleman, and M. S. Strano, Nat. Nano 7, 699 (2012).

[2] K. F. Mak, C. Lee, J. Hone, J. Shan, and T. F. Heinz, Phys. Rev. Lett. 105, 136805 (2010).

[3] C. Lee, H. Yan, L. E. Brus, T. F. Heinz, J. Hone, and S. Ryu, ACS Nano 4, 2695 (2010).

[4] H. Sahin, S. Tongay, S. Horzum, W. Fan, J. Zhou, J. Li, J. Wu, and F. M. Peeters, Phys. Rev. B 87, 165409 (2013).

[5] S. Tongay, H. Sahin, C. Ko, A. Luce, W. Fan, K. Liu, J. Zhou, Y.-S. Huang, C.-H. Ho, J. Yan et al., Nat. Commun. 5, 3252 (2014).

[6] H. Zhao, J. Wu, H. Zhong, Q. Guo, X. Wang, F. Xia, L. Yang, P. Tan, and H. Wang, Nano Res. 8, 3651 (2015).

[7] K. Friemelt, M. Ch. Lux Steiner, and E. Bucher, J. Appl. Phys. 74, 5266 (1993).

[8] C. H. Ho, M. H. Hsieh, C. C. Wu, Y. S. Huang, and K. K. Tiong, J. Alloys Compd. 442, 245 (2007).
[9] D. A. Chenet, O. B. Aslan, P. Y. Huang, C. Fan, A. M. van der Zande, T. F. Heinz, and J. C. Hone, Nano Lett. 15, 5667 (2015).

[10] K. K. Tiong, C. H. Ho, and Y. S. Huang, Solid State Commun. 111, 635 (1999).

[11] E. Liu, Y. Fu, Y. Wang, Y. Feng, H. Liu, X. Wan, W. Zhou, B. Wang, L. Shao, C.-H. Ho et al., Nat. Commun. 6, 6991 (2015).

[12] J. Gao, L. Li, J. Tan, H. Sun, B. Li, J. C. Idrobo, C. V. Singh, T.-M. Lu, and N. Koratkar, Nano Lett. 16, 3780 (2016).

[13] M. Chhowalla, H. S. Shin, G. Eda, L.-J. Li, K. P. Loh, and H. Zhang, Nat. Chem. 5, 263 (2013).

[14] M. Kertesz and R. Hoffmann, J. Am. Chem. Soc. 106, 3453 (1984).

[15] K. Friemelt, L. Kulikova, L. Kulyuk, A. Siminel, E. Arushanov, C. Kloc, and E. Bucher, J. Appl. Phys. 79, 9268 (1996).

[16] C. H. Ho, P. C. Liao, Y. S. Huang, and K. K. Tiong, Phys. Rev. B 55, 15608 (1997). 
[17] L. Hart, S. Dale, S. Hoye, J. L. Webb, and D. Wolverson, Nano Lett. 16, 1381 (2016).

[18] X. Ling, S. Huang, E. H. Hasdeo, L. Liang, W. M. Parkin, Y. Tatsumi, A. R. T. Nugraha, A. A. Puretzky, P. M. Das, B. G. Sumpter et al., Nano Lett. 16, 2260 (2016).

[19] J. Augustin, V. Eyert, T. Boker, W. Frentrup, H. Dwelk, C. Janowitz, and R. Manzke, Phys. Rev. B 62, 10812 (2000).

[20] M. Bovet, S. van Smaalen, H. Berger, R. Gaal, L. Forró, L. Schlapbach, and P. Aebi, Phys. Rev. B 67, 125105 (2003).

[21] J. M. Riley, F. Mazzola, M. Dendzik, M. Michiardi, T. Takayama, L. Bawden, C. Granerd, M. Leandersson, T. Balasubramanian, M. Hoesch et al., Nat. Phys. 10, 835 (2014).

[22] L. Bawden, S. P. Cooil, F. Mazzola, J. M. Riley, L. J. CollinsMcIntyre, V. Sunko, K. W. B. Hunvik, M. Leandersson, C. M. Polley, T. Balasubramanian et al., Nat. Commun. 7, 11711 (2016).

[23] G. Kresse and J. Hafner, Phys. Rev. B 47, 558 (1993).

[24] G. Kresse and J. Hafner, Phys. Rev. B 49, 14251 (1994).

[25] G. Kresse and J. Furthmuller, Phys. Rev. B 54, 11169 (1996).

[26] G. Kresse and J. Furthmuller, Comput. Mater. Sci. 6, 15 (1996).

[27] G. Kresse and D. Joubert, Phys. Rev. B 59, 1758 (1999).

[28] J. P. Perdew, A. Ruzsinszky, G. I. Csonka, O. A. Vydrov, G. E. Scuseria, L. A. Constantin, X. Zhou, and K. Burke, Phys. Rev. Lett. 100, 136406 (2008)

[29] J. P. Perdew, K. Burke, and M. Ernzerhof, Phys. Rev. Lett. 77, 3865 (1996).

[30] W. Travis, C. E. Knapp, C. N. Savory, A. M. Ganose, P. Kafourou, X. Song, Z. Sharif, J. K. Cockcroft, D. O. Scanlon, H. Bronstein et al., Inorg. Chem. 55, 3393 (2016).

[31] A. M. Ganose, K. T. Butler, A. Walsh, and D. O. Scanlon, J. Mater. Chem. A 4, 2060 (2016).

[32] A. V. Krukau, O. A. Vydrov, A. F. Izmaylov, and G. E. Scuseria, J. Chem. Phys. 125, 224106 (2006).

[33] A. E. Maughan, A. M. Ganose, M. M. Bordelon, E. M. Miller, D. O. Scanlon, and J. R. Neilson, J. Am. Chem. Soc. 138, 8453 (2016).

[34] C. N. Savory, A. M. Ganose, W. Travis, R. S. Atri, R. G. Palgrave, and D. O. Scanlon, J. Mater. Chem. A 4, 12648 (2016).
[35] D. Hobbs, G. Kresse, and J. Hafner, Phys. Rev. B 62, 11556 (2000).

[36] V. N. Stroco, J. Electron Spectrosc. Relat. Phenom. 130, 65 (2003).

[37] W. Ku, T. Berlijn, and C.-C. Lee, Phys. Rev. Lett. 104, 216401 (2010).

[38] S.-H. Lee, G. Xu, W. Ku, J. S. Wen, C. C. Lee, N. Katayama, Z. J. Xu, S. Ji, Z. W. Lin, G. D. Gu et al., Phys. Rev. B 81, 220502 (2010).

[39] Y. F. Nie, P. D. C. King, C. H. Kim, M. Uchida, H. I. Wei, B. D. Faeth, J. P. Ruf, J. P. C. Ruff, L. Xie, X. Pan et al., Phys. Rev. Lett. 114, 016401 (2015).

[40] P. Li and I. Appelbaum, Phys. Rev. B 92, 195129 (2015).

[41] J. Yeh and I. Lindau, At. Data Nucl. Data Tables 32, 1 (1985).

[42] O. B. Aslan, D. A. Chenet, A. M. van der Zande, J. C. Hone, and T. F. Heinz, ACS Photon. 3, 96 (2016).

[43] P. D. C. King, T. D. Veal, and C. F. McConville, Phys. Rev. B 77, 125305 (2008).

[44] P. D. C. King, T. D. Veal, D. J. Payne, A. Bourlange, R. G. Egdell, and C. F. McConville, Phys. Rev. Lett. 101, 116808 (2008).

[45] P. D. C. King, R. C. Hatch, M. Bianchi, R. Ovsyannikov, C. Lupulescu, G. Landolt, B. Slomski, J. H. Dil, D. Guan, J. L. Mi et al., Phys. Rev. Lett. 107, 096802 (2011).

[46] M. S. Bahramy, P. D. C. King, A. de la Torre, J. Chang, M. Shi, L. Patthey, G. Balakrishnan, P. Hofmann, R. Arita, N. Nagaosa et al., Nat. Commun. 3, 1159 (2012).

[47] M. M. Ugeda, A. J. Bradley, S.-F. Shi, F. H. da Jornada, Y. Zhang, D. Y. Qiu, W. Ruan, S.-K. Mo, Z. Hussain, Z.-X. Shen et al., Nat. Mater. 13, 1091 (2014).

[48] J. M. Riley, W. Meevasana, L. Bawden, M. Asakawa, T. Takayama, T. Eknapakul, T. K. Kim, M. Hoesch, S.-K. Mo, H. Takagi et al., Nat. Nanotechnol. 10, 1043 (2015).

[49] M. Gehlmann, I. Aguilera, G. Bihlmayer, S. Nemsak, P. Nagler, P. Gospodaric, G. Zamborlini, M. Eschbach, V. Feyer, F. Kronast et al., arXiv:1702.04176.

[50] J. L. Webb, L. S. Hart, D. Wolverson, C. Chen, J. Avila, and M. C. Asensio, arXiv:1704.06042. 